Supplement of Atmos. Chem. Phys., 17, 8453-8471, 2017

https://doi.org/10.5194/acp-17-8453-2017-supplement

(C) Author(s) 2017. This work is distributed under

the Creative Commons Attribution 3.0 License.

(c) (1)

Supplement of

\title{
Diffusivity measurements of volatile organics in levitated viscous aerosol particles
}

\section{Sandra Bastelberger et al.}

Correspondence to: Sandra Bastelberger (sandra.bastelberger@env.ethz.ch)

The copyright of individual parts of the supplement might differ from the CC BY 3.0 License. 
The Table below gives the retrieved data for PEG-4 diffusivity in ternary aqueous solutions of sucrose and PEG-4 as shown in Figs 7, 8 and A2 of the manuscript.

\begin{tabular}{|l|l|l|l|l|l|}
\hline $\mathrm{T}[\mathrm{K}]$ & $\begin{array}{l}\text { mol- } \\
\text { fraction } \\
\text { sucrose }\end{array}$ & aw & $\begin{array}{l}\mathrm{D}(\mathrm{PEG}-4) \\
{\left[\mathrm{cm}^{2} \mathrm{~s}^{-1}\right]}\end{array}$ & $\begin{array}{l}\text { uncertainty } \\
\text { range }+ \text { (+) } \\
{\left[\mathrm{cm}^{2} \mathrm{~s}^{-1}\right]}\end{array}$ & $\begin{array}{l}\text { uncertainty } \\
\text { range }(-) \\
{\left[\mathrm{cm}^{2} \mathrm{~s}^{-1}\right]}\end{array}$ \\
\hline 292.65 & 0.481 & 0.26 & $3 \mathrm{E}-15$ & $1.2 \mathrm{E}-15$ & $8.6 \mathrm{E}-16$ \\
\hline 292.65 & 0.443 & 0.30 & $6.2 \mathrm{E}-14$ & $2.5 \mathrm{E}-14$ & $1.8 \mathrm{E}-14$ \\
\hline 292.65 & 0.418 & 0.34 & $2.45 \mathrm{E}-13$ & $2 \mathrm{E}-13$ & $7 \mathrm{E}-14$ \\
\hline 292.65 & 0.382 & 0.39 & $1.29 \mathrm{E}-12$ & $5.1 \mathrm{E}-13$ & $3.7 \mathrm{E}-13$ \\
\hline 292.65 & 0.342 & 0.44 & $8 \mathrm{E}-12$ & $4.2 \mathrm{E}-11$ & $3 \mathrm{E}-12$ \\
\hline 288.15 & 0.446 & 0.29 & $4 \mathrm{E}-15$ & $1.1 \mathrm{E}-15$ & $1.6 \mathrm{E}-15$ \\
\hline 288.15 & 0.413 & 0.33 & $3.8 \mathrm{E}-14$ & $1.1 \mathrm{E}-14$ & $1.5 \mathrm{E}-14$ \\
\hline 288.15 & 0.394 & 0.35 & $1.1 \mathrm{E}-13$ & $3.1 \mathrm{E}-14$ & $4.4 \mathrm{E}-14$ \\
\hline 288.15 & 0.376 & 0.38 & $3.5 \mathrm{E}-13$ & $1 \mathrm{E}-13$ & $1.4 \mathrm{E}-13$ \\
\hline 283.15 & 0.393 & 0.35 & $1 \mathrm{E}-14$ & $2.9 \mathrm{E}-15$ & $4 \mathrm{E}-15$ \\
\hline 283.15 & 0.362 & 0.39 & $1.2 \mathrm{E}-13$ & $3.4 \mathrm{E}-14$ & $8 \mathrm{E}-14$ \\
\hline 283.15 & 0.354 & 0.42 & $3.4 \mathrm{E}-13$ & $1 \mathrm{E}-13$ & $2 \mathrm{E}-13$ \\
\hline
\end{tabular}

